\title{
Assessment of Dry Eye Syndrome among Computer Programmers in Computer Training Institutes in Benin City
}

\author{
Ayishetu Oshoke Shuaibu ${ }^{1}$, Sarah Ikhuemose Ebuwa ${ }^{1}$, and Abimbola Esther Ikuemonisan ${ }^{1}$ \\ ${ }^{1}$ University of Benin, Faculty of Life Sciences, Department of Optometry.
}

\begin{tabular}{l|l|l} 
Corresponding author: Ayishetu Oshoke Shuaibu & Email: ayishetu.garuba@uniben.edu & Phone: +2348055644890
\end{tabular}

\begin{abstract}
Purpose: The purpose of this study was to evaluate dry eye syndrome among computer programmers in computer training institutes in Benin City.

Methods: This cross-sectional study was carried out using 250 participants of 18 years and above who were computer instructors and students of various computer training institutes in Benin City. Sociodemographic data and relevant ocular history were obtained from the participants. External and Internal examinations of the eyes were carried out to check for the presence of any ocular abnormalities. Dry eye disease was assessed subjectively with the Ocular Surface Disease Index (OSDI) questionnaire and objectively by performing Schirmer's test and Tear Break-up Time (TBUT). Participants with an OSDI score of $\geq 35$ and who had $<10 \mathrm{~mm}$ to Schirmer's I test or $<10$ secs in Tear break-up Time (TBUT) test were considered to have dry eye.
\end{abstract}

Results: The mean age of the participants was $25.32 \pm 7.38$ years and $68 \%$ were males. The prevalence of dry eye among the computer programmers was found to be $30.8 \%$. Mean TBUT and Mean Schirmer's Test value for those with dry eye was $10.21 \mathrm{~s} \pm 3.44 \mathrm{~s}$ and $12.46 \pm 7.27 \mathrm{~mm}$ respectively. This study showed that age $(p=0.213)$, gender $(p=0.243)$ and duration of time spent on computer display units $(p=0.124)$ were not statistically significant determinants of dry eye among the computer programmers.

Conclusion: Computer programmers are predisposed to developing dry eye diseases. It is therefore imperative for them to go for regular eye examination to prevent ocular surface disorders and therefore maintain comfortable vision.

Keywords: Dry Eye, Schirmer Test, Tear Break-up Time, Ocular Surface Disease Index, Computer Programmer.

\section{Introduction}

Dry eye is a common disease estimated to have affected 25 to 30 million people all over the world ${ }^{1,2}$.
Males and females have been associated with the risk of developing dry eye disease, but females are considered to be at a greater risk due to deficient

Patil S, Trivedi H, Jethva J. Evaluation of dry eye in computer users. International Journal of community medicine and public health, 2016; 3(12): 3403-3407.

Phadatare SP, Momin M, Nighojkar P, Askarkar S, Singh KK. A comprehensive review on dry eye disease: diagnosis, medical management, recent developments, and future challenges Adv pharm. 2015;2015:1-12 
tear secretion from estrogen deficiency due to increasing age $\mathrm{e}^{3,4}$.

Dry Eye Syndrome is a multifactorial disease of the ocular surface characterized by a loss of homeostasis of the tear film and accompanied by ocular symptoms in which tear film instability and hyperosmotic, ocular surface inflammation, damage and neurosensory abnormalities play etiological roles 5 . The normal tear film is made up of 3 layers (the lipid layer, aqueous layer and mucin layer). The lipid layer which helps to prevent evaporation is the outer most layer. The middle layer which is also known as the aqueous layer consists of $90 \%$ water and the innermost layer, the mucin layer which helps to bind the tear film to the epithelium. A disruption in any of these layers such as tear deficiency or excessive tear evaporation could cause damage to the ocular surface and this is associated with a variety of symptoms reflecting ocular discomfort ${ }^{6}$. Dry eye therefore occurs when people don't have enough tears or correct composition of tears on the surface of their eyes which results in discomfort. Predisposing factors of dry eye include; advanced age, gender, certain medications, environment, workplace stress (arid atmosphere, constant wind currents), prolonged use of video display screens, and presence of contact lens ${ }^{7}$.

A Computer Programmer is a skilled professional who codes, tests, debugs and maintains the comprehensive instructions known as computer programs that devices should follow to execute their functions ${ }^{8}$. Computer Programmers use computers for long hours. Extended visual tasking during computer use, television watching and prolonged reading could provoke the symptoms of dry eye disease ${ }^{9}$. Computer use for long hours in a day has been reported as a significant risk factor for Dry eye disease. This has been attributed to the decrease in blink rate while using these devices, therefore hampering the uniform distribution of the tear film over the ocular surface ${ }^{10}$. The cause of the associated eye discomfort complained by computer users have been linked to decreased mucin concentration which is a lubricant in the eye ${ }^{10}$. Akkaya, et al. ${ }^{11}$ conducted a study to evaluate the effects of long-term computer use on tear production and evaporation. He reported that long term computer usage may cause evaporative type of dry eye disease. Also, another study on the spectrum of dry eye disease in computer users in Manipur, India was conducted by Shamurailatapam, et al. ${ }^{12}$ on 600 computer users, they concluded that the prevalence of dry eye increases with increasing duration of computer use. This study is therefore aimed at evaluating dry eye syndrome among computer programmers, thereby determining the prevalence of dry eye among computer users and also the relationship between dry eye syndrome and age, gender and duration spent on the computer.

\section{Methods}

This study was a cross sectional study, conducted in computer training institutes in Benin City which

\footnotetext{
Majumder M, Manna B, Devi M. A. Clinical study on prevalence of dry eye disease among the patients attending O.P.D in a tertiary care hospital in Assam, India. International Journal of Science

Ranjan R, Shukla K, Singh V, Mishra N, Sinhas S, Sharma B. Prevalence of dry eye and its association with various risk factors in rural setup of western Uttar Pradesh in a tertiary care hospital. Open Journal of Preventive Medicine 2016;6: 57-63.

Craig JP, Nelson DJ, Azar DT, Belmonte C, Bron AJ, Sunil KC, et. al. Report of the definition and classification subcommittee of the international dry eye workshop (DEWS-2017). Ocula surface 2017;15(4): 802-812.

Lemp MA, Baudouin C, Baum J. The definition and classification of dry eye disease: report of the definition and classification subcommittee of the international dry eye workshop. Ocula Surface 2007; 5: 75-92.

Sharma B. Dry Eye: Demography and attributable risk factors. Medical Journal. 2011; 11: 16-22.

Tecchopedia - the IT Educational Site. Computer Programmer. https://www.techopedia.com/definition/6589/computer-programmer

Schlote T, Kadner G, Freudenthaler N. Marked reduction and distinct patterns of eye blinking in patients with moderately dry eyes during video display terminal use. Graefe's Archive for Clinica and Experimental Ophthalmology 2004:242(4):306-12.

Uchino M, Uchino Y, Dogru M, Kwawastima M. Dry eye diseases and work productivity loss in visual display terminals users: the Osaka study. American Journal of Ophthalmology $2014 ; 157$

Akkaya S, Atakan T, Acikalin B, Aksoy S, Ozkurt Y. Effects of long term computer use on eye dryness. North Clin Istranb 2018; 5(4): 319-322.

12. Shamurailatapam S S, Das R, Devi S. Spectrum of dry eye disease in computer users of Manipur India. International scholarly and scientific research and innovation. 2017 ; 11 (4): 194-197.
} 
include Mikon Institute of Information, Institute for Continuing Education (ICE), Complete Computers and Technology Limited.

The sample size was determined using the single population proportion formula. Prevalence of 50\%, $95 \%$ confidence interval, $7 \%$ margin of error and $20 \%$ anticipated non-response was considered. This gave a sample size of 245 that was rounded up to 250 .

A total of 250 participant aged 18 years and above who were selected randomly from different computer training institutes in Benin City were included in this study. The three computer schools where selected from different areas in Benin City. Participants were selected randomly from each of the three computer schools. 100 participants were randomly selected from Mikon institute of Information, 80 from Institute of Continuing Education and 70 participants from Complete Computers and Technology Limited. The study was conducted in accordance with the Helsinki Declaration. Ethical clearance was obtained from the University of Benin ethical committee and the management of the various computer training institutes in Benin City (ethical approval number.: ECUBEN $\backslash$ LSC.OPT/19/039). Verbal consent was also obtained from all subjects.

The study included students and instructors of the computer training institutes, 18 years and above, who use computer for more than 2 hours daily for the past 6 months, Subjects were with no known systemic or ocular disease and have not had any resent ocular surgical management, not on any topical medication and do not use contact lenses.

A brief history was obtained from the participants with an emphasis on the history of dry eye. Socio- demographic data obtained from the participants include; Subject's Age, Gender, Systemic condition and history of contact lens use. External Examination was carried out using penlight and magnifier to check for the presence of any ocular surface abnormality and adnexa abnormalities. The internal examination was carried out using a Keeler Ophthalmoscope to check for the presence of any abnormalities.

Subjects who fulfilled the inclusion criteria were administered the Ocular Surface Disease Index (OSDI) questionnaire. The OSDI questionnaire had a total of 12 questions centered on dry eyes. These were subdivided into 3 sections. The first section covered symptoms of dry eye, while the second was on the effects of dry eye and the third section was on aggravating symptoms. Tear quality and quantity were then evaluated using tear breakup time test and Schirmer I test respectively.

In the Schirmer I test, the strip was placed in the lateral one-third of the lower eyelid. The subject then closed their eyes for 5 minutes. After 5 minutes, the strip was removed and the level of wetting (in millimeters) was noted ${ }^{13}$. Readings less than 10 millimeters wetting was considered as a diagnostic of dry eye ${ }^{14}$.

Tear breakup time is the interval between the last complete blink and the first appearance of a dry spot, or disruption of the tear film ${ }^{5}$ Tear Break up Time TBUT was assessed by asking the subject to blink a few times before the examination. Fluorescein was then applied to the lower part of the bulbar conjunctiva surface and the subject was told to blink to spread the fluorescein. The tear film was assessed using a burton lamp and the interval from the last blink to the appearance of the first dry eye

\footnotetext{
Reshma BKS Iram S. Prevalence of dry eye in computer users. IP Internaltional Journal of Ocular Oncology and Oculoplasty 2020;6(2):95-98

Bron A, Abelson M, Ousler G, Tomlinson A, Yokoi NN, Smith JA, et al. Methodologies to diagnose and monitor dry eye disease: report of the diagnostic methodology subcommittee of the international dry eye workshop. Ocular Surface. 2007;5(2): 108-23.
} 
spot on the cornea noted. Tear film break up time measuring less than 10 seconds was considered as a diagnostic of dry eye ${ }^{14}$.

Data obtained was analyzed using Statistical Package for Social Scientist (SPSS) and Descriptive analysis such as percentages, and tables were used to summarize the data. Chi square was used to analyse the relationship between dry eye and age, gender and duration of computer square. Level of significance was set at $\mathrm{p} \leq 0.05$. Using Craig et al., ${ }^{5}$ criteria, subjects with an OSDI score of $\geq 35$ and who had $<10 \mathrm{~mm}$ to Schirmer's I test or $<10$ secs in Tear break-up Time (TBUT) test were considered to have dry eye. Some subjects had OSDI score $\geq 35$ and good tear quality (TBUT) but poor tear quantity (Schirmer I test) and some had OSDI score $\geq 35$ but had good tear quantity(Schirmer I test) but poor tear quality (TBUT) and were considered dry eye subjects. We found the mean Schirmer I test and TBUT results of all dry eye patients. Hence the mean of both tests was slightly higher than the cut off.

\section{Results}

This study profiled the prevalence of dry eye syndrome, relationships between age, gender and duration of computer use and dry eye syndrome among participants. The study comprised of both male and female subjects within the age group 18 years and above. The mean age of the participants was $25.32 \pm 7.38$ years. The prevalence of dry eye among the computer users was found to be $30.8 \%$ . (Table 1).

Table 1: Demographic data of computer Programmers

Frequency $n=250 \quad$ Percentage $(\%)$

Age group (years)

$<30$

214

85.6

$31-40$

25

$41-50$

7

$51-60$

4

Gender

Female

80

170

Male

Duration of computer use

$<2$

$2-5$

49

$6-9$

123

$>=10$
32.0

19.6

49.2

10.0

2.8

1.6

68.0

29.2

\footnotetext{
Craig JP, Nelson DJ, Azar DT, Belmonte C, Bron AJ, Sunil KC, et. al. Report of the definition and classification subcommittee of the international dry eye workshop (DEWS-2017). Ocular surface 2017;15(4): 802-812

Bron A, Abelson M, Ousler G, Tomlinson A, Yokoi NN, Smith JA, et al. Methodologies to diagnose and monitor dry eye disease: report of the diagnostic methodology subcommittee of the international dry eye workshop. Ocular Surface. 2007;5(2): 108-23.
} 


\section{Dry Eye Status}

Non dry eye

( Mean TBUT $=14.13 \mathrm{~s} \pm 3.15 \mathrm{~s}$,

Mean Schirmer's Test value

$=19.93 \mathrm{~mm} \pm 4.56 \mathrm{~mm}$ )

Dry eye

( Mean TBUT $=10.21 \mathrm{~s} \pm 3.44 \mathrm{~s})$

Mean Schirmer's Test value $=12.46$

$\pm 7.27$

Total

Total mean TBUT $=12.92 \pm 3.15$

Total mean Schirmer's test value $=$

$17.63 \pm 6.51$

Table 2: Relationship between age, gender, duration of computer use and dry eye syndrome

\begin{tabular}{lccc}
\multicolumn{5}{c}{ Group } & & P-value \\
\hline Age group & Non-dry eye $(\%)$ & Dry eye (\%) & Total \\
$\leq 30$ & $153(71.5)$ & $61(28.5)$ & $214(100)$ \\
$31-40$ & $15(60)$ & $10(40)$ & $25(100)$ \\
$41-50$ & $3(43)$ & $4(57)$ & $7(100)$ \\
$51-60$ & $2(50)$ & $2(50)$ & $4(100)$ \\
Total & $173(69.2)$ & $77(30.8)$ & $250(100)$ \\
Gender & & & \\
Female & $53(66.3)$ & $27(33.7)$ & $80(100)$ \\
Male & $120(70.6)$ & $50(29.4)$ & $170(100)$ \\
Total & $173(69.2)$ & $77(30.8)$ & $250(100)$ \\
Duration of computer use(hours) & & & \\
$<2$ & $2(40)$ & $3(60)$ & 5.243 \\
$2-5$ & $34(69.4)$ & $15(30.6)$ & $49(100)$ \\
$6-9$ & $92(74.8)$ & $31(25.2)$ & $123(100)$ \\
$\geq 10$ & $45(61.6)$ & $28(38.4)$ & $73(100)$ \\
Total & $173(62.2)$ & $77(30.8)$ & $250(100)$
\end{tabular}

Table 2 shows there was no statistically significant association between age of computer operators and the Dry eye syndrome $\left(\mathrm{X}^{2}=4.493, \mathrm{P}=0.213\right)$. It also shows there was no significant association between the gender and the dry eye syndrome $\left(\mathrm{X}^{2}=2.832, \mathrm{P}=0.243\right)$. There was no statistically significant relationship between duration of computer use and the prevalence of dry eye syndrome among the participants $\left(\mathrm{X}^{2}=\right.$ 5.764, $\mathrm{P}=0.124)$. 


\section{Discussion}

The purpose of this research was to evaluate dry eye syndrome among computer programmers. Dry eye syndrome is a significant and serious disorder of the tear film and ocular surface.

In different population-based studies, the prevalence of dry eye among computer users have been estimated to vary from $25 \%^{1}$ to $75 \%{ }^{15}$. In this study, the prevalence of dry eye was $30.8 \%$ which was consistent with previous studies ${ }^{1,16-19}$. A study conducted by Patil, et al., ${ }^{1}$ in a hospital-based study among computer users in India, the prevalence of dry eye disease was found to be $25 \%$. Kwashima, et al. ${ }^{16}$ designed a web-based screening for dry eye disease among computer users and found the prevalence of dry to be $32.2 \%$. Mansoori, et al. ${ }^{17}$ also evaluated dry eye syndrome and associated risk factors among computer users in Karachi, Pakistan and found the prevalence of dry eye to be $28 \%$. A study also by Hagan et al. ${ }^{18}$ on non-contact lens computer operators found $68 \%$ men and $73 \%$ women reported dry eye symptoms. A prevalence of $44.3 \%$ was reported by Asiedu, et al. ${ }^{19}$ in a study on symptomatic dry eye and its associated factors. They however used only questionnaires (SPEED and OSDI) for evaluation of symptomatic dry eye in their study.

In this study as shown in table 2, there was no significant association between the age of subjects and dry eye syndrome $(\mathrm{p}=0.213)$. This is similar to the study carried out by Akkaya, et al. ${ }^{11}$ where there was no significant association between age and dry eye $(p>0.05)$. Dry eye was however found to be significantly associated with age group above 40 years in a study conducted by Olaniyan, et $a .^{20}$ in an adult population in South-West Nigeria. The age distribution in this study showed that the majority of subjects fell within the age range of less than 30 years (214 subjects). This might be the reason why the age of the subjects had no significant association with dry eye as a larger percentage of the study population was less than 30 years. The mean age of this study was $25.32 \pm 7.38$ years.

The gender distribution of this study (table 1) had a higher percentage of males (68\%) and a lesser percentage of females (32\%). Table 2 showed no significant difference between the gender of subjects and dry eye syndrome $(\mathrm{p}=0.243)$. The study carried out by Akkaya, et al. ${ }^{11}$ also reported no significant difference between gender and dry eye syndrome ( $>0.05$ ). In a study carried out by Majumder, et al. ${ }^{3}$, it was discovered that the majority of females with dry eye syndrome were postmenopausal women. Majority of females in this study were not among this post-menopausal group.

The mean TBUT score of Akkaya, et al. ${ }^{11}$ study on effects of prolonged computer use on eye dryness (11.37 \pm 3.69 seconds) was close to the mean TBUT score (12.92 \pm 3.15 seconds) of this study. The majority of subjects in this study as seen in table 2 had TBUT values lesser than 10 seconds (a poorer stability of tears). Long term use of computer causes instability in the distribution of tears on the ocular surface, which could lead to evaporation of the teardrops (evaporative dry eye syndrome) ${ }^{11} \mathrm{Gajta}$, et $a l .{ }^{21}$ also reported this, that working on the

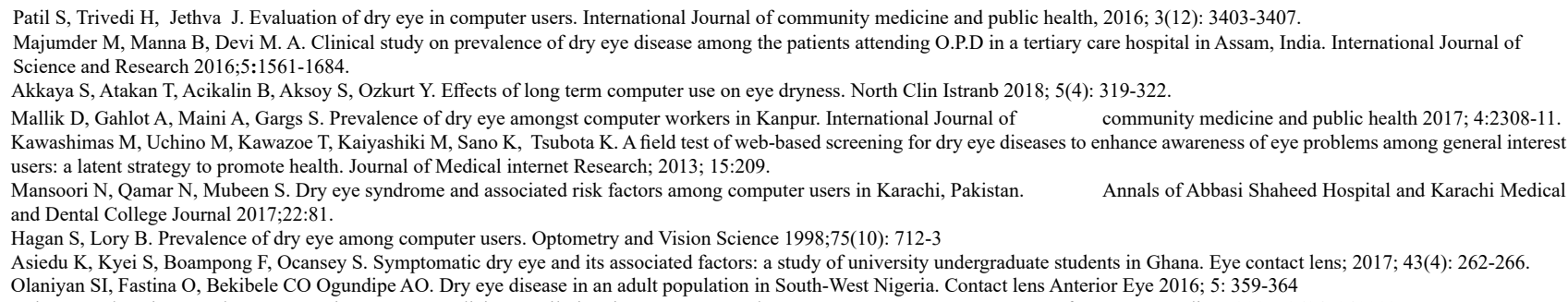


computer for the long term affects tear film stability and quality of tears which leads to lower values of TBUT and eventually leads to dry eye syndrome.

In this study, although it was noticed that a longer time spent on the computer predisposed subjects to dry eye syndrome. This was not statically significant $(\mathrm{p}=0.124)$.

The Schirmer's mean value $(17.63 \pm 6.51 \mathrm{~mm})$ in this study is consistent with Akkaya, et al. ${ }^{12}$ study on effects of prolonged computer use on eye dryness where the Schirmer's mean value was $16.80 \pm 2.04 \mathrm{~mm}$. It is however slightly different from the Schirmer's mean value of Unlu, et al. ${ }^{22}$, study on "the comparison of ocular surface disease index questionnaire, tear film break up time and Schirmer's tests for the evaluation of the tear film in computer users with and without symptomatology" where they had a mean value of $25.80 \pm 8.43 \mathrm{~mm}$.

Studies on dry eye diagnosis are still going on and because tear film components are related getting a sensitive method to diagnose dry eye is still difficult. However, we used the OSDI questionnaire to evaluate dry eye symptoms and evaluated tear quality and quantity using tear breakup time and schirmer's test respectively to ascertain our dry eye subjects. The study shows that prevalence of dry eye is on the rise especially among computer programmers.

\section{Conclusion}

The study revealed the prevalence of dry eye syndrome among computer programmers was $30.8 \%$. The study also showed that spending longer hours with the computer could reduce tear break up time. The prevalence of dry eye among computer programmers is increasing and so awareness among computer users is needed to encourage regular eye examination and thereby preventing ocular surface disorders and maintaining comfortable vision. 